\title{
Article \\ Fabrication of Large Area, Ordered Nanoporous Structures on Various Substrates for Potential Electro-Optic Applications
}

\author{
Hongsub Jee ${ }^{1}$, Kiseok Jeon ${ }^{2}$, Min-Joon Park ${ }^{2}$ and Jaehyeong Lee ${ }^{1, *(1)}$ \\ 1 Department of Electrical and Computer Engineering, Sungkyunkwan University, Suwon 16419, Korea; \\ hsjee@skku.edu \\ 2 Smart Energy \& Nano R\&D Group, Korea Institute of Industrial Technology, Gwangju 61012, Korea; \\ jks8287@kitech.re.kr (K.J.); pk5659@kitech.re.kr (M.-J.P.) \\ * Correspondence: jaehyeong@skku.edu
}

check for

updates

Citation: Jee, H.; Jeon, K.; Park, M.-J.; Lee, J. Fabrication of Large Area, Ordered Nanoporous Structures on Various Substrates for Potential Electro-Optic Applications. Appl. Sci. 2021, 11, 12136. https://doi.org/ 10.3390/app112412136

Academic Editors: Fabrice Goubard, Branko Kolaric, André Stefanov and Brana Jelenkovic

Received: 31 October 2021

Accepted: 15 December 2021

Published: 20 December 2021

Publisher's Note: MDPI stays neutral with regard to jurisdictional claims in published maps and institutional affiliations.

Copyright: (c) 2021 by the authors. Licensee MDPI, Basel, Switzerland. This article is an open access article distributed under the terms and conditions of the Creative Commons Attribution (CC BY) license (https:// creativecommons.org/licenses/by/ $4.0 /)$.

\begin{abstract}
Nanoporous structures have attracted great attention in electronics, sensor and storage devices, and photonics because of their large surface area, large volume to surface ratio, and potential for high-sensitivity sensor applications. Normally, electron or ion beam patterning can be used for nanopores fabrication by direct writing. However, direct writing is a rather expensive and time-consuming method due to its serial nature. Therefore, it may not translate to a preferred manufacturing process. In this research, a perfectly ordered large-area periodic pattern in an area of approximately $1 \mathrm{~cm}^{2}$ has been successfully fabricated on various substrates including glass, silicon, and polydimethylsiloxane, using a two-step process comprising visible light-based multibeam interference lithography and subsequent pattern transfer processes of reactive ion etching and nanomolding. Additionally, the multibeam interference lithography templated anodized aluminum oxide process has been described. Since the fabrication area in multibeam interference lithography can be extended by using a larger beam size, it is highly cost effective and manufacturable. Furthermore, although not described here, an electrodeposition process can be utilized as a pattern transfer process. This large-area perfectly ordered nanopore array will be very useful for high-density electronic memory and photonic bandgap and metamaterial applications.
\end{abstract}

Keywords: multibeam interference lithography (MIL); reactive ion etching (RIE); large area; nanopore; transfer; pattern

\section{Introduction}

Because of their large surface area, large volume to surface ratio, and potential for high sensitivity sensor applications, nanoporous structures have drawn great attention in electronics, sensor and storage devices, and photonics [1-6]. Electron or ion beam patterning can be used for nanopore fabrication by direct writing [7-10]. However, direct writing is a rather expensive and time-consuming method due to its serial nature. Therefore, it may not translate to a preferred manufacturing process. Meantime, metal anodization processes, i.e., anodized aluminum oxide (AAO) and anodized titanium oxide (ATO) processes, are used for large-area nanopore templates [11-14], but their array patterns tend to contain locally disordered portions, which would limit their usage for electronic memory devices or photonic devices requiring perfectly ordered arrays over a large area. Multibeam interference lithography (MIL), however, could be a good candidate for the patterning of perfectly ordered large-area photonic crystals and can be applied for photonic, electronic applications [15-19]. MIL can form interference patterns on photosensitive polymer. In our previous work, MIL and two-photon lithography were combined by two-step exposures and a single development process. This new approach, adding patterns on the repeated nanoporous structures, suggested a new path from restricted usages of nanoporous structures to various applications but essentially, the newly fabricated structures were still made of the same material, polymer, and there was a limit to their 
usage for diverse applications [20]. To overcome its limitations and construct a functional electronic or photonic device, pattern transfer to the underneath layers or substrates is required.

In this work, three-beam interference lithography with a visible light source is used to form hexagonal symmetric interference patterns on a large photoresist (PR) layer. Subsequently, various pattern transfer processes to different substrates such as reactive ion etching (RIE) to glass and Si substrates, nanomolding to a polydimethylsiloxane (PDMS) substrate, and anodization to an aluminum-coated substrate were performed. By combining MIL and RIE/nanomolding, nanoporous polymer structures could be transferred to various substrates and this can provide the opportunity to be used for a variety of applications for electronic and photonic devices.

\section{Materials and Methods}

To fabricate nanoporous structures with a large area, multibeam interference lithography was performed using a cw Nd:YVO4 laser (Verdi V6, Coherent, Palo Alto, CA, USA) with a wavelength of $532 \mathrm{~nm}$. The whole covered area by MIL was approximately $1 \mathrm{~cm}^{2}$ and the covered area can be adjusted by varying the aperture size of the beam. As PR, Epon SU-8 (Miller-Stephenson Chemical, Inc., Sylmar, CA, USA) was selected due to its good solubility and transparency in visible and UV light. The SU-8 was doped with a photosensitizer, Rubrene (Sigma-Aldrich, Inc., Burlington, MA, USA) and a photoacid generator, diaryliodonium hexafluoroantimonate (PC-2506, Polyset, Mechanicville, NY, USA) to respond to the green laser. Figure 1 shows the experimental setup and a schematic of three-beam interference lithography where the incident angle of each beam was $45^{\circ}$ from the vertical line, and the phase difference was $120^{\circ}$ between beams in the horizontal plane. Depending on the phase angle of each incident beam and the PR type, either a hexagonal symmetric nanopore array or a nanopillar array was formed.

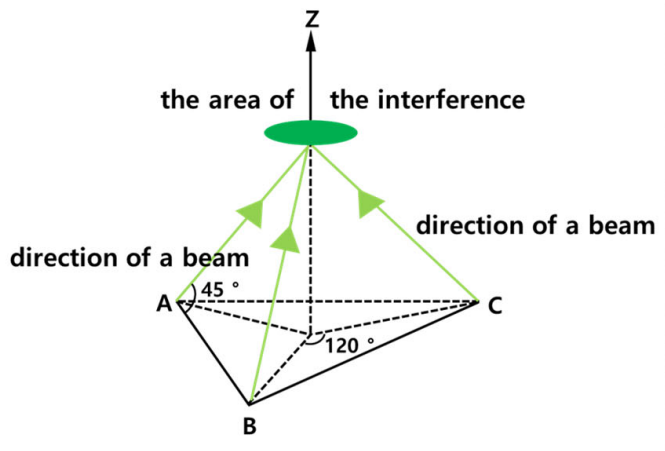

(a)

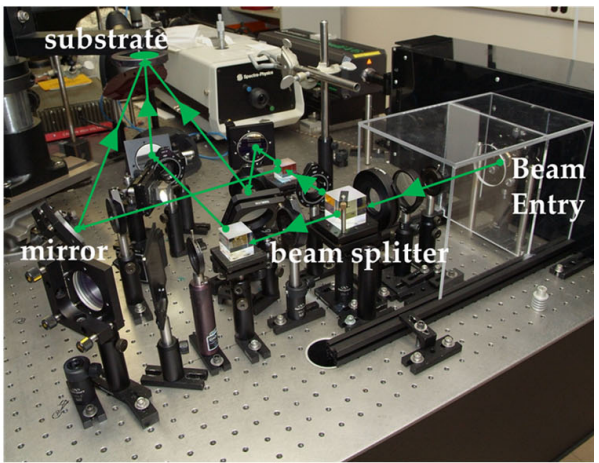

(b)

Figure 1. Schematic of three beam interference (a). Experimental setup of MIL system (b).

Figure 2 shows the nanoporous structures on the substrate fabricated by MIL. As is shown, well-ordered interference patterns were fabricated, which covered $1 \mathrm{~cm}^{2}$. Depending on the optical property of the substrate, a buffer layer was used between the substrate and the photopatterning layer to minimize the reflection. 


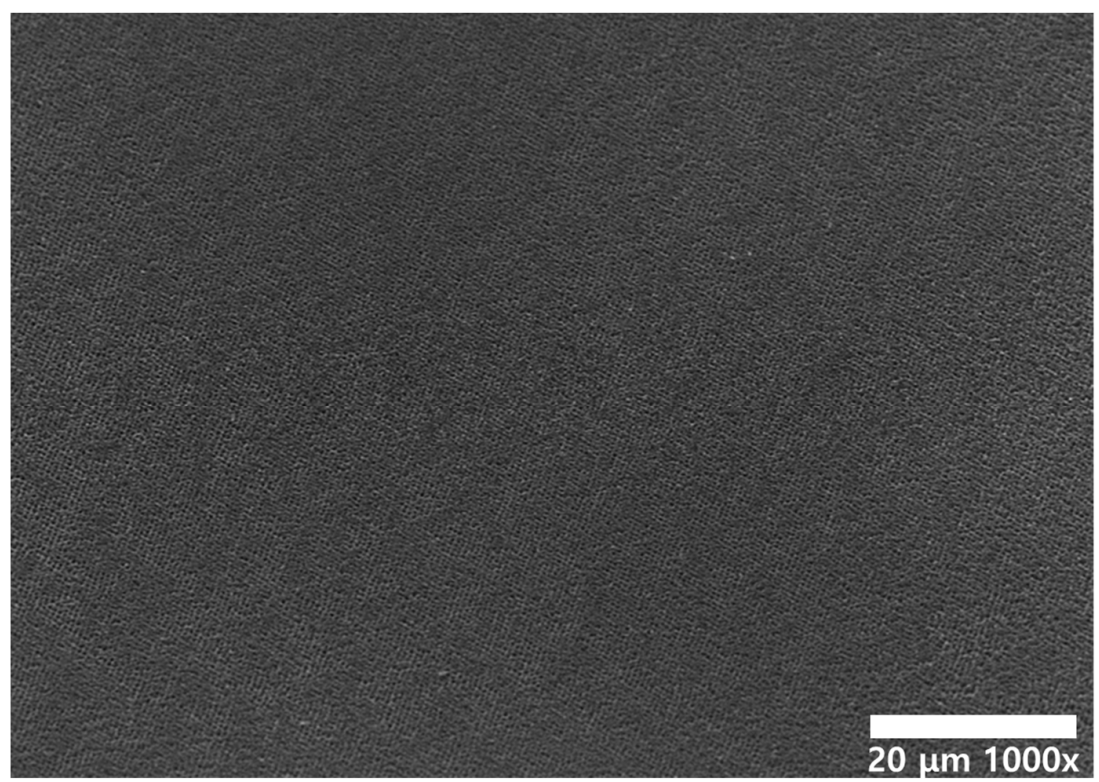

Figure 2. Large-area $\left(1 \mathrm{~cm}^{2}\right)$ covered nanoporous structures patterned by MIL.

\section{Results and Discussion}

As pattern transfer methods, three different approaches are shown in Figure 3. Figure 3a used a (RIE) process for a pattern transfer method to a Si or glass substrate. When glass is used as a substrate, the buffer layer between the PR and the substrate may not be critical since the refractive index difference between SU-8 $(n=1.59)$ and glass $(n=1.50)$ is relatively small and no significant portion of optical dose is reflected to generate parasitic patterns. On the other hand, when $\mathrm{Si}$ is used, reflection between SU-8 and $\mathrm{Si}(n=3.42)$ would be quite significant due to its large refractive index difference, and the buffer layer design becomes very critical. SU-8 doped with Rhodamine B (RhB) dye (Sigma-Aldrich, Inc., Burlington, MA, USA) has been used because of its strong absorption of green light and high quantum efficiency. First, RhB was dissolved in cyclopentanone and mixed with SU-8 and photoacid generator, where the weight ratio of SU-8:RhB:PC was 10:0.1:1. It was spin-coated on a polished Si substrate at $2000 \mathrm{rpm}$ for $40 \mathrm{~s}$. After soft baking it on a hotplate at $95^{\circ} \mathrm{C}$ for a minute, it was flood-exposed under the multiband UV light for one minute and hard baked at $160^{\circ} \mathrm{C}$ for $10 \mathrm{~min}$. The resultant thickness was approximately $2.5 \mu \mathrm{m}$. Next, a SU-8 PR layer with a weight ratio of SU-8:Rubrene:PC of 100:0.2:2.5 was spin-coated at $4000 \mathrm{rpm}$ for $40 \mathrm{~s}$, followed by soft baking at $95{ }^{\circ} \mathrm{C}$ for a minute (Figure 3(a1)). Three-beam interference lithography was performed on a circular exposure area with a diameter of $1 \mathrm{~cm}$. The cumulative intensity of the exposure energy was $0.6 \mathrm{~W} \mathrm{~cm} \mathrm{~cm}^{-2}$ and exposed beams for 3 to $8 \mathrm{sec}$ gave the total exposure dosage for 2 to $5 \mathrm{~J} \mathrm{~cm}^{-2}$. When the incident beams reached the PR layer, which has very good thermal stability, the exposed areas increased in molecular weight and photochemically transformed to form insoluble products [21]. The specimen was post baked on a hot plate at $95^{\circ} \mathrm{C}$ for a minute and cooled down to room temperature, followed by development in propylene glycol monomethyl ether acetate (PGMEA). The thickness was approximately $300-400 \mathrm{~nm}$ (Figure 3(a2)). RIE was performed in an oxygen-dominant environment for buffer layer patterning (Figure 3(a3)) and in a chlorine-dominant environment for Si layer patterning (Figure 3(a4)). Figure 4 shows a fabricated photonic crystal membrane at the stage depicted in Figure 3(a2). 
1
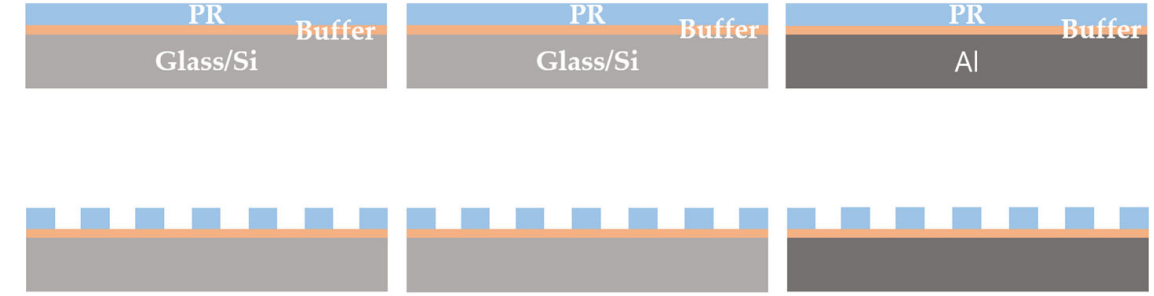

3

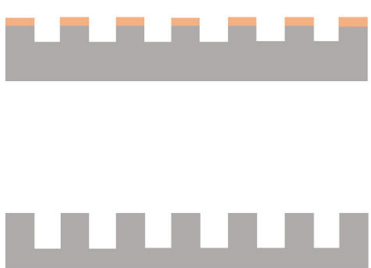

(a)
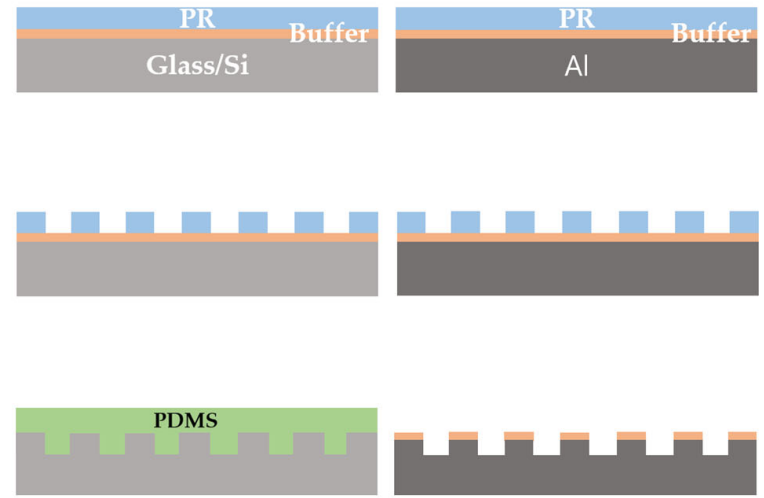

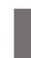

(b)

Figure 3. Various pattern transfer processes: (a) Reactive ion etching, (b) Nanomolding, (c) Anodization; 1 Deposition materials on the different substrates, 2 Etching PR layer, 3 Pattern transference, 4 Final structures.

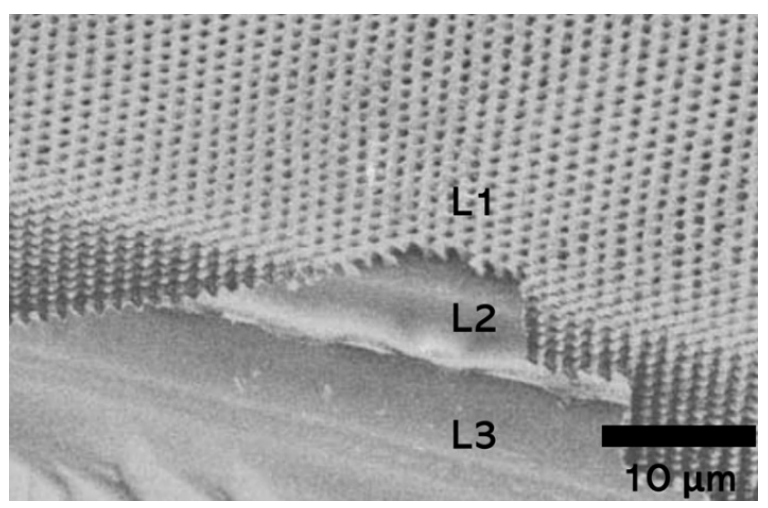

Figure 4. Various pattern transfer processes: Fabricated photonic crystal membrane: L1-PR, L2-Buffer, L3-Si (refer to Figure 3(a2)) on etching.

The three layers are clearly seen with the top layer patterned in a hexagonal symmetric shape. Figure 5 shows a pattern transferred glass substrate, where RIE has been performed in $\mathrm{O}_{2}: \mathrm{CF}_{4}$ of $15: 3.5 \mathrm{sccm}$ with a power of $20 \mathrm{~W}$ for $30 \mathrm{~s}$ for buffer layer etching and in $\mathrm{O}_{2}: \mathrm{CH}_{4}$ of $5: 40 \mathrm{sccm}$ with $70 \mathrm{~W}$ for $2 \mathrm{~min}$ for glass etching [13]. Figure $3 \mathrm{~b}$ shows a pattern transfer process using polydimethylsiloxane (PDMS) nanomolding. After the interference lithography and RIE process with PR on the glass substrate to make nanoporous glass structures as shown in Figure 3a, PDMS was poured on the nanoporous glass surface and cured at $60^{\circ} \mathrm{C}$ for $2 \mathrm{~h}$. After cooling down to room temperature, a nanopillar array, which is the negative form of the nanopore array, was formed upon separation. Figure $6 \mathrm{a}$ is the top view of a perfectly ordered hexagonal symmetric nanopore array, with the inset showing a magnified oblique view. Figure $6 \mathrm{~b}$ shows a replicated PDMS structure after nanomolding, with the inset showing a magnified view of the pillar array. The scanning electron microscopy image demonstrates good fidelity. The anodized aluminum oxide (AAO) process converts pure aluminum into aluminum oxide in a specific shape, as shown in Figure 7, when it is anodized in an acidic environment under a bias voltage. The pore size and the pitch between pores can be controlled by the magnitude of the bias voltage and the type of acids. For example, the pore pitch is in the range of 50-60 nm at a bias voltage of $19-25 \mathrm{~V}$ in sulfuric acid $\left(\mathrm{H}_{2} \mathrm{SO}_{4}\right), 220 \mathrm{~nm}-300 \mathrm{~nm}$ at $120-250 \mathrm{~V}$ in oxalic acid $\left(\mathrm{H}_{2} \mathrm{C}_{2} \mathrm{O}_{2}\right)$, 
and $405-500 \mathrm{~nm}$ at $160-195 \mathrm{~V}$ in phosphoric acid $\left(\mathrm{H}_{3} \mathrm{PO}_{4}\right)$. If the bias voltage is lower than the optimal range, pores formed are not well ordered. If the bias voltage is higher than the optimal range, the barrier layer breaks down and substrate burning occurs. Fabricated anodized aluminum oxide layer samples are shown in Figure 8. A two-step AAO process has been used to form more uniform pore sizes. First, aluminum foil undergoes thermal pretreatment at $400{ }^{\circ} \mathrm{C}$ for one hour, which forms a thin layer of oxide layer, preventing initial breakdown upon applying bias voltage. The first anodization is performed in oxalic acid at $40 \mathrm{~V}$ for $6 \mathrm{~min}$, which gives rise to relatively random pores, as shown in Figure $8 \mathrm{a}$. The first anodized layer is etched away using a solution consisting of $1.8 \mathrm{wt} \%$ chromic acid and $6 \mathrm{wt} \%$ phosphoric acid for an hour. After etching, small bumps are formed along the barrier depicted in Figure 7. The bumps serve as guides for the second anodization. The second anodization is performed in oxalic acid at $40 \mathrm{~V}$ for $4 \mathrm{~min}$. Optionally, pore widening step is performed using $6 \mathrm{wt} \%$ phosphoric acid for an hour. Figure $8 \mathrm{~b}, \mathrm{c}$ are the cross-section view and the top view, respectively. The average pore size and the average pitch are $60 \mathrm{~nm}$ and $100 \mathrm{~nm}$, respectively [15]. Figure 3c shows a direct pattern transfer onto an aluminum layer using AAO immediately followed by MIL. The anodization is being performed using phosphoric acid at $160 \mathrm{~V}$ to have a pore pitch of $500 \mathrm{~nm}$. As an alternative to the direct anodization process after MIL, template-assisted anodization can be used. However, there are some challenges, including reflection and pore size matching issues between one from MIL and one from AAO. In order to preserve the perfect pore ordering from MIL, further work is required.

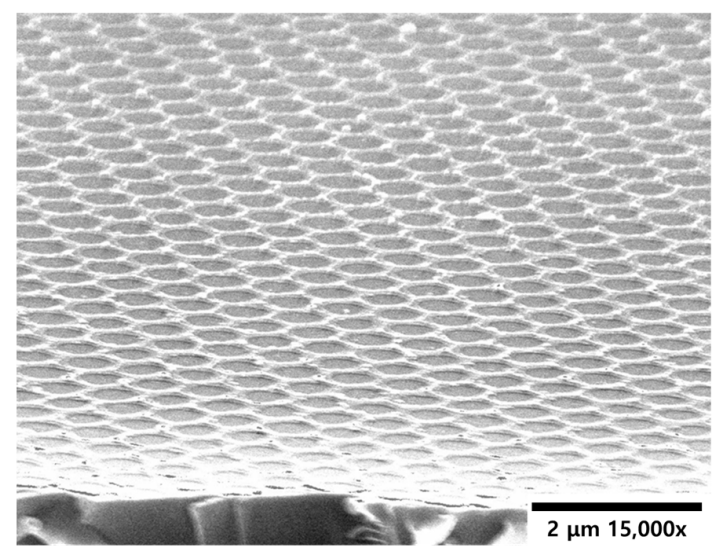

Figure 5. Transferred pattern on a glass substrate.

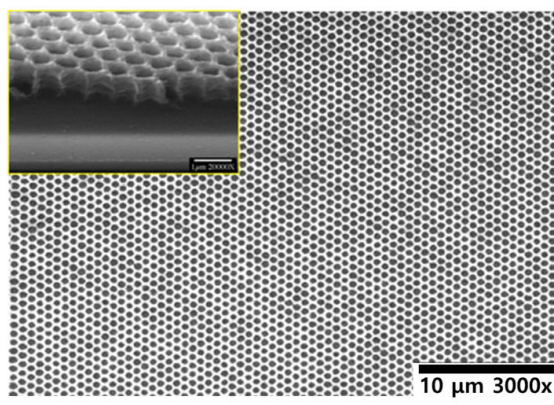

(a)

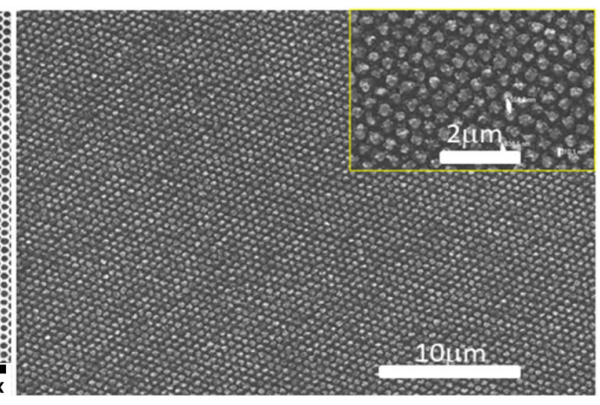

(b)

Figure 6. Perfectly ordered large-area nanopore array: (a) Top view of nanopore array (inset shows a magnified oblique view), (b) Top view of nanomolded PDMS pillar array (inset shows a magnified view). 


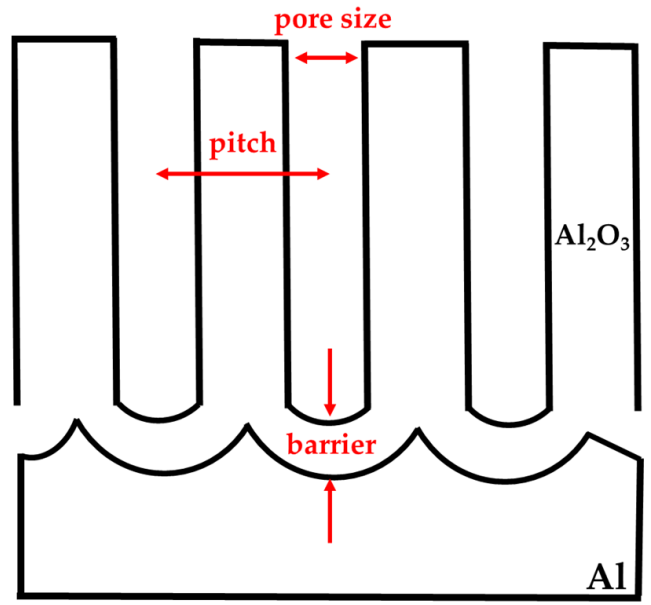

Figure 7. Schematic of the cross-section of anodized aluminum oxide.

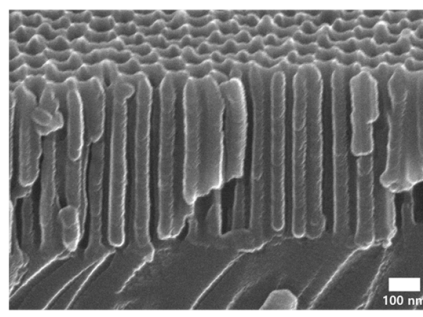

(a)

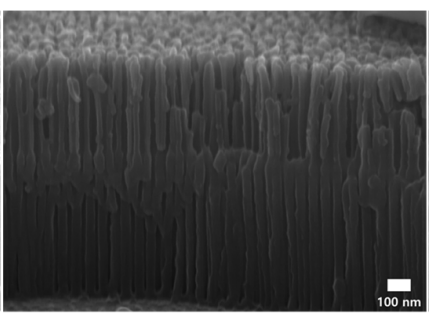

(b)

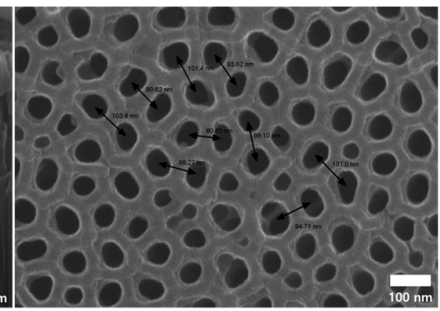

(c)

Figure 8. SEM images of fabricated anodized aluminum oxide layers: (a) After the first anodization, (b) After the second anodization, (c) Top view of the second anodization sample.

\section{Conclusions}

A perfectly ordered large-area periodic pattern in an area of approximately $1 \mathrm{~cm}^{2}$ has been successfully fabricated on various substrates including glass, $\mathrm{Si}$, and PDMS, using a two-step process comprising visible light-based MIL and subsequent pattern transfer processes of RIE and nanomolding. Additionally, the MIL-templated AAO process has been described. Since the fabrication area in MIL can be extended by using a larger beam size, it is highly cost effective and manufacturable. This large-area perfectly ordered nanopore array will be very useful for high-density electronic memory and photonic bandgap and metamaterial applications, sensors, nanolens and photonics [22].

Author Contributions: Conceptualization, H.J.; methodology, H.J. and K.J.; data curation, H.J. and M.-J.P. writing_original draft preparation, H.J.; writing—review and editing, H.J. and J.L.; supervision, J.L. All authors have read and agreed to the published version of the manuscript.

Funding: This work was supported by the Technology Innovation Program funded by the Ministry of Trade, Industry \& Energy (MOTIE) of the Republic of Korea. (No. 20010965).

Institutional Review Board Statement: Not applicable.

Informed Consent Statement: Not applicable.

Data Availability Statement: The data that support the findings of this study are available upon reasonable request.

Conflicts of Interest: The authors declare no conflict of interest. 


\section{References}

1. Deyang, J.; Tao, L.; Harald, F. Patterning and applications of nanoporous structures in organic electronics. Nano Today 2020, 31, 100843.

2. Huang, Y.G.; Shiota, Y.; Wu, M.Y.; Su, S.Q.; Yao, Z.S.; Kang, S.; Kanegawa, S.; Li, G.-L.; Wu, S.-Q.; Kamachi, T.; et al. Superior thermoelasticity and shape-memory nanopores in a porous supramolecular organic framework. Nat. Commun. 2016, 7, 11564. [CrossRef] [PubMed]

3. Mohsin, A.B.; Jun, K.; Hoyoung, J.; Seok-Min, K. Fabrication of Highly Packed Plasmonic Nanolens Array Using Polymer Nanoimprinted Nanodots for an Enhanced Fluorescence Substrate. Polymers 2018, 10, 649.

4. Li, X.; Shao, J.; Tian, H.; Li, X.; Chen, X.; Zhu, X. High-transmittance and focal controllable plano-convex lenses with embedded nanolens bottoms formed by electrowetting on a colloidal monolayer. J. Mater. Chem. C 2020, 8, 2659-2663. [CrossRef]

5. Junsoo, P.; Changhoon, C. Aluminum based reflective nanolens arrays to improve the effectiveness of ultraviolet inactivation of Escherichia coli O157:H7 and Listeria monocytogenes in water and a sucrose solution. Food Sci. Biotechnol. 2020, 29, $1281-1287$.

6. Sergey, A.S.; Alexey, Z.; Dmitri, V.P.; Stanislav, O.G.; Eugeny, V.P.; Alexey, P.P.; Svetlana, N.K.; Sergei, A.K.; John, B.B.R.; Sergey, I.K.; et al. Plasmonic Nanolenses Produced by Cylindrical Vector Beam Printing for Sensing Applications. Sci. Rep. 2019, 9, 19750.

7. Cai, J.; Lv, C.; Hu, C.; Luo, J.; Liu, S.; Song, J.; Shi, Y.; Chen, C.; Zhang, Z.; Ogawa, S.; et al. Laser direct writing of heteroatom-doped porous carbon for high-performance micro-supercapacitors. Energy Storage Mater. 2020, 25, 404-415. [CrossRef]

8. Rebecca, L.W.; Michael, J.B.; Beecher, H.W.; Elizabeth, R.K.; Mark, A.F.; Richard, J.M.; Gery, L.M. Direct writing of textured ceramics using anisotropic nozzles. J. Eur. Ceram. Soc. 2021, 41, 1945-1953.

9. Sergey, G.; Vitaliy, A.G.; Joan, V.-C.; Christian, D. Direct e-beam writing of dense and high aspect ratio nanostructures in thick layers of PMMA for electroplating. Nanotechnology 2010, 21, 295303-295310.

10. Birgitta, S.; Loic, A.; Laurent, B.; Abdelghani, O.; Madouri, A.; Gilles, P.; Juan, P.; Ralf, J.; Jacques, G. Direct FIB fabrication and integration of "single nanopore devices" for the manipulation of macromolecules. Microelectron. Eng. 2010, 87, 1300-1303.

11. Zhang, W.; Tian, Q.; Chen, Z.; Zhao, C.; Chai, H.; Wu, Q.; Li, W.; Chen, X.; Deng, Y.; Song, Y. Arrayed nanopore silver thin films for surface-enhanced Raman scattering. RSC Adv. 2020, 10, 23908-23915. [CrossRef]

12. Alexander, M.H.; Pravini, S.F.; Smilgies, D.M.; Jeffrey, M.M. Freeing Organic Semiconductor Nanowires from Nanoporous Aluminum Oxide Templates: Effects on Morphology, Crystal Structure, and Molecular Aggregation. Cryst. Growth Des. 2021, 21, 721-728.

13. Yu, J.; Wang, F.; Wang, Y.; Gao, H.; Li, J.; Wu, K. Interfacial reaction growth approach to preparing patterned nanomaterials and beyond. Chem. Soc. Rev. 2010, 39, 1513-15325. [CrossRef]

14. Shobha, S.; Kyoung-Tae, K.; Alexander, B.; Natalia, M.L.; Paras, N.P. Fabrication and Characterization of Gold-Polymer Nanocomposite Plasmonic Nanoarrays in a Porous Alumina Template. ACS Nano 2010, 4, 2249-2255.

15. $\mathrm{Hu}, \mathrm{X}$; Z Zhang, X.; Chen, X.; Luo, M. Solution route to large area all- $\mathrm{TiO}_{2}$ one-dimensional photonic crystals with high reflectivity and different structural colors. Nanotechnology 2020, 31, 135209. [CrossRef]

16. Menaka, D.Z.; Masahiro, Y.; Bongshik, S.; Kenji, I.; Takuya, I.; Shumpei, K.; Koki, I.; Yoshinori, T.; Ranko, H.; John, G.; et al. Thermal management for CW operation of large-area double-lattice photonic-crystal lasers. J. Opt. Soc. Am. B 2020, 37, 3882-3887.

17. Hyuk Moon, J.; Yang, S. Creating Three-Dimensional Polymeric Microstructures by Multi-Beam Interference Lithography. J. Macromol. Sci. C 2005, 45, 351-373. [CrossRef]

18. Woo, L.; Ran, J.; Ulrich, G.; Kornelius, N. Fast fabrication of long-range ordered porous alumina membranes by hard anodization. Nat. Mater. 2006, 5, 741-747.

19. Baquedano, E.; Torné, L.; Caño, P.; Postigo, P.A. Increased Efficiency of Solar Cells Protected by Hydrophobic and Hydrophilic Anti-Reflecting Nanostructured Glasses. Nanomaterials 2017, 7, 437. [CrossRef] [PubMed]

20. Hongsub, J.; Min-Joon, P.; Kiseok, J.; Chaehwan, J.; Jaehyeong, L. Combining Interference Lithography and Two-Photon Lithography for Fabricating Large-Area Photonic Crystal Structures with Controlled Defects. Appl. Sci. 2021, 11, 6559.

21. Marc, J.M. Lithography. In Fundamentals of Microfabrication, 2nd ed.; CRC Press: Boca Raton, FL, USA, 2002 ; pp. 2-10.

22. Woo, L.; Ran, J.; Caroline, A.R.; Ulrich, G.; Kornelius, N. Wafer-scale Ni imprint stamps for porous alumina membranes based on interference lithography. Small 2006, 2, 978-982. 